\title{
Novel Monte Carlo scheme for systems with short-ranged interactions
}

\author{
Georgios C. Boulougouris and Daan Frenkel ${ }^{\mathrm{a})}$ \\ Fundamenteel Onderzoek der Materie (FOM) Institute for Atomic and Molecular Physics, Kruislaan 407, \\ 1098 SJ Amsterdam, The Netherlands
}

(Received 28 January 2005; accepted 19 April 2005; published online 29 June 2005)

\begin{abstract}
We propose a Monte Carlo (MC) sampling algorithm to simulate systems of particles interacting via very short-ranged discontinuous potentials. Such models are often used to describe protein solutions or colloidal suspensions. Most normal MC algorithms fail for such systems because, at low temperatures, they tend to get trapped in local potential-energy local minima due to the short range of the pair potential. To circumvent this problem, we have devised a scheme that changes the construction of trial moves in such a way that the potential-energy difference between initial and final states drops out of the acceptance rule for the Monte Carlo trial moves. This approach allows us to simulate systems with short-ranged attraction under conditions that were unreachable up to now. () 2005 American Institute of Physics. [DOI: 10.1063/1.1931652]
\end{abstract}

\section{INTRODUCTION}

Simulations of molecular systems where the particles are interacting via short-range potentials are interesting for two reasons. On the one hand, these models can be used to describe systems of considerable practical importance, e.g., colloidal suspensions and protein solutions, where the constituent particles attract each other strongly when they are close to contact. On the other hand, the study of such models is of interest in its own right, as it is clear that the shape of the phase diagram of spherical particles depends strongly on the range of the intermolecular forces. ${ }^{1-5}$ An example is the existence of a metastable liquid-liquid transition in model systems with short-ranged attractions. ${ }^{6-10}$ Strictly speaking, the interaction between (macro) molecules in solution is not given by an interaction potential, but by an interaction free energy - the potential of mean force. ${ }^{11,12}$ Metastable liquiddemixing transition has been observed experimentally in solutions of globular proteins, and may play an important role in the pathway for protein crystallization. ${ }^{13-17}$

In what follows, we focus on the simplest model for a system with short-ranged attractive interactions, namely, the square-well fluid. Before discussing our algorithm, it is useful to analyze why simulations of square-well fluids with short-ranged interactions are problematic. Consider a fluid of particles with a hard-core diameter $\sigma$ and an attractive well with depth $\varepsilon$ and width $\delta \sigma$. The second virial coefficient of such a fluid is given by

$$
B_{2}=\frac{2 \pi \sigma^{3}}{3}\left[1-\left(e^{\beta \varepsilon}-1\right)\left((1+\delta)^{3}-1\right)\right]
$$

where $\beta=1 / k T$. It is clear that, if the well is narrow $(\delta \ll 1)$, the effect of attraction only becomes noticeable if $\exp (\beta \varepsilon)$ $\gg 1$. This is the "interesting" regime where, for instance, liquid-liquid demixing can occur. However, the fact that $\exp (\beta \varepsilon) \gg 1$ makes conventional Monte Carlo (MC) sam-

\footnotetext{
a) Author to whom correspondence should be addressed. Fax: +31-206684106. Electronic mail: frenkel@amolf.nl
}

pling difficult. If two particles are within the range of the attraction, the acceptance probability of a trial move that moves the two particles outside that range becomes very small $[\exp (-\beta \varepsilon)]$. Similarly, the reverse move is unlikely because the pair attraction is only active in a volume of order $\delta \ll 1$. This problem becomes worse if we consider the interaction of a particle with $n$ other particles. Then the relevant Boltzmann factor is $\exp (n \beta \varepsilon)$, where $n$ may be as large as 12 . The net result is that "bond-breaking" moves are virtually never accepted and "bond-making" moves are virtually never attempted. This problem can be overcome by changing the construction of the Markov chain in the Monte Carlo algorithm. Before we explain this in more detail, let us once more consider a system of square-well particles with a shortranged attraction with well depth $\varepsilon$. Now consider that we insert a trial particle in this system. Of the total volume $V$, only a subvolume $V_{a}$ is accessible (i.e., free of hard-core overlaps). We can now decompose this volume in a part $V_{0}$ where the trial particle does not interact with any other particle. A part $V_{1}$ corresponds to all points where the trial particle interacts with only one other particle. Similarly, we can define volumes $V_{2}, V_{3}, \ldots, V_{n}$ where a particle interacts with $1,2, \ldots, n$ other particles. Of course, these different volumes have different Boltzmann weights $P_{B}(n) \propto V_{n} e^{n \beta \varepsilon}$. If we can somehow evaluate $V_{n}$, then we can perform a Monte Carlo scheme where trial moves go from a random point in subvolume $V_{m}$ to a random point in subvolume $V_{n}$. The probability to perform such a trial move is determined by the ratio $\left(V_{m} e^{m \beta \varepsilon}\right) /\left(V_{n} e^{n \beta \varepsilon}\right)$. This approach is at the basis of the algorithm used in Refs. 1 and 2 to simulate adhesive hard spheres (i.e., a square-well fluid in the limit $\delta \rightarrow 0$ ). However, the approach used in Refs. 1 and 2 is only valid for $\delta \rightarrow 0$. Here we consider the general case where $\delta \neq 0$. Several authors have devised algorithms to improve the MC sampling of systems with such short-ranged interactions. ${ }^{18,19}$ These methods are based on biasing the attempt to perform bondbreaking and bond-making moves, and recover detailed balance through the appropriate unbiasing factor in the acceptance criteria. In this manner Wierzchowski and Kofke ${ }^{19}$ 


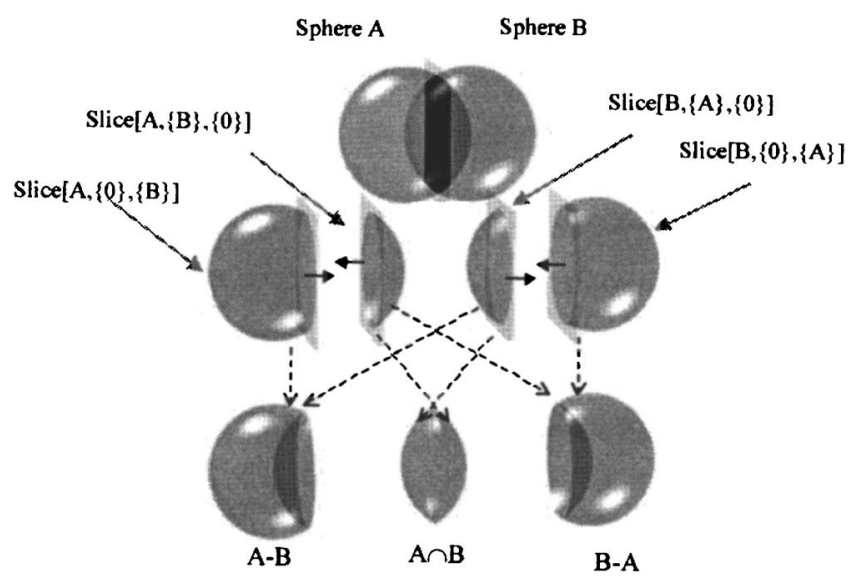

FIG. 1. Schematic representation of the reduction of the intersectiondifference-union calculation in a problem of intersecting spheres with planes.

constructed a bias move where the bias is used to determine the probability of attempting to move a particle that does not interact with any of each neighbors, to a trial position where it is interacting with at least a single other particle, and vice versa (possible interactions with more than one particles, even overlaps accounted for, not in the trial probability, but in the acceptance criterion). These algorithms are simple and of great practical use. However, they are applicable to a limited range of $\delta$ values since the probability of selecting state where a particle interacts with many neighbors tends to be small, while its Boltzmann weight is large. Moves in and out of such regions are unlikely. In the algorithm discussed below, no such limitation exists. However, the price that we pay is that we have to sacrifice the simplicity of the algorithm.

\section{APPROACH}

Dod and Theodorou ${ }^{20}$ have developed a general algorithm that yields a closed expression for the total volume and the exposed area of a union of $n$ spheres of arbitrary size, delimited by a set of planes. In the present work, we extend the algorithm of Ref. 20 to the calculation of the volume of intersection and the volume of difference of spheres with arbitrary size. The basic idea behind our approach is that we can then use the inclusion-exclusion principle to compute all the volumes $\left(V_{0}, V_{1}, V_{2}, \ldots, V_{12}\right)$.

As with the evaluation of the union of spheres, the first step is to decompose the problem into a problem of a single sphere intersected by a set of planes. For every pair of overlapping spheres, a plane may be drawn perpendicular to the line connecting the two sphere centers (Fig. 1). When computing the union of these spheres the plane removes a cup from both spheres. In contrast, when evaluating the intersection of the same pair of spheres, we need to know the volume of the cups. Finally, in the case of calculating the difference of two spheres the cup of the first sphere should be removed whereas for the subtracted sphere the cup remains and the rest of the sphere is removed. Which part of the sphere will be removed is defined by the direction of the normal vector of the plane. According to the convention used by Dod and
Theodorou $^{20}$ a plane removes the part of the sphere that lies on the side pointed by the normal vector. Each of the two spheres is given a copy of the plane of intersection, including a unit normal vector directed so that it points out of the remaining part of the sphere, as shown in Fig. 1. Note that the unit vectors in the two spheres are pointing in opposite directions in the case of union and intersection calculations whereas in the case of calculating differences the unit vectors are identical (Fig. 1). In this way the spheres are completely decoupled from each other and, therefore, can be analyzed separately and, moreover, the same sphere can participate simultaneously in an arbitrary number of union-intersectiondifference calculations with other spheres. This procedure is repeated for all sphere pairs in the system. In addition, some of the spheres in the system may be delimited by one or more of the external planes defined in the problem statement (see below). Thus we only have to consider the truncation of all spheres in the system by sets of planes. The calculation then continues in exactly the same manner as in the case of the union calculation. ${ }^{20}$ As in the case of the union calculation the method is exact for any arbitrary number of overlapping sphere and sphere sizes and is only subject to numerical precision.

In order to describe the proposed algorithm the following function is introduced:

$$
\text { Slice }[I_{i} ; \underbrace{\left\{I_{i \neq i}, \ldots\right\}}_{n} ; \underbrace{\left\{I_{k \neq i . i}, \ldots\right\}}_{m}]
$$

as the remaining volume of sphere $I_{i}$ after slicing the sphere with the $n$ intersection planes formed by the intersection of sphere $I_{i}$ and each of the spheres $I_{j}$ with normal vector equal to $\mathbf{r}_{I_{i}}-\mathbf{r}_{I_{j}} /\left\|\mathbf{r}_{I_{i}}-\mathbf{r}_{I_{j}}\right\|$ (forming a cup), and $m$ intersection planes formed by the intersection of sphere $I_{i}$ and each of the spheres $I_{k}$ with normal vector equal to $-\left(\mathbf{r}_{I_{i}}-\mathbf{r}_{I_{k}}\right) /\left\|\mathbf{r}_{I_{i}}-\mathbf{r}_{I_{k}}\right\|$ (removing a cup). For the sake of simplicity we shall refer to the former ones as forming-a-cup (FC) planes, whereas the latter as removing-a-cup (RM) planes. The Slice function depends only on the position and radius of sphere $I_{i}$, and on the intersecting planes of sphere $I_{i}$ with the remaining spheres. In this way the spheres are decoupled and the "truncated" volume of each sphere can be calculated directly from the analytical geometrical algorithm. ${ }^{20}$ For the Monte Carlo algorithm, we only consider moves inside a small, cubic volume, the "trial box." In our calculations, we also consider the (possible) intersections of each sphere with the six bounding planes of the trial box. For simplicity, we do not mention these planes separately in what follows.

As illustrated in Fig. 1 from the definition of the Slice function it follows that

$$
\begin{aligned}
& V_{A \cup B}=\operatorname{Slice}[A ;\{\varnothing\} ;\{B\}]+\operatorname{Slice}[B ;\{\varnothing\} ;\{A\}], \\
& V_{A \cap B}=\operatorname{Slice}[A ;\{B\} ;\{\varnothing\}]+\operatorname{Slice}[B ;\{A\} ;\{\varnothing\}], \\
& V_{A-B}=\operatorname{Slice}[A ;\{\varnothing\} ;\{B\}]-\operatorname{Slice}[B ;\{A\} ;\{\varnothing\}], \\
& V_{B-A}=\operatorname{Slice}[B ;\{\varnothing\} ;\{A\}]-\operatorname{Slice}[A ;\{B\} ;\{\varnothing\}] .
\end{aligned}
$$

In the case where volume $A$ is completely embedded in volume $B$ it follows that 
$V_{A \cup B}=V_{B}, \quad$ Slice $[A ;\{\varnothing\} ; B]=0, \quad$ Slice $[B ;\{\varnothing\} ; A]=V_{B}$,

$V_{A \cap B}=V_{A}, \quad$ Slice $[A ;\{B\} ;\{\varnothing\}]=V_{A}, \quad$ Slice $[B ;\{A\} ;\{\varnothing\}]$

$=0$.

For the case of $n$ spheres:

$$
\begin{aligned}
& V_{I_{1} \cup I_{2} \cup I_{3} \cup I_{4} \cup . . \cup I_{n}}=\sum_{i=1}^{n} \operatorname{Slice}[I_{i} ;\{\varnothing\} ; \underbrace{\left\{I_{j \neq i, \ldots}\right\}}_{n-1}], \\
& V_{I_{1} \cap I_{2} \cap I_{3} \cap I_{4} \cap \ldots \cap I_{n}}=\sum_{i=1}^{n} \operatorname{Slice}[I_{i} ; \underbrace{\left\{I_{j \neq i}, \ldots\right\}}_{n-1} ;\{\varnothing\}] \text {, } \\
& V_{i_{i}-(\underbrace{}_{1} \cup \ldots\left(U I_{i+i} U \ldots\right)} \underbrace{}_{m} \\
& =\operatorname{Slice}\left[I_{i} ;\{\varnothing\} ;\{\underbrace{\left.J_{1}, \ldots, J_{i \neq i}, \ldots\right\}}_{m}\}\right] \\
& -\sum_{j}^{m} \operatorname{Slice}[J_{j \neq i} ;\left\{I_{i}\right\} ; \underbrace{\left\{\ldots, J_{k \neq j, i}, \ldots\right\}}_{m-1}\}, \\
& \underbrace{V_{\left(I_{1} \cap \ldots \cap I_{j} \cap \ldots\right)}-(\underbrace{\left.J_{1} \cup \ldots \cup J_{j} \cup \ldots\right)})}_{n} \\
& \left.=\sum_{i}^{n} \operatorname{Slice}[I_{i} ; \underbrace{\ldots, I_{j \neq i}, \ldots}_{n-1}\} ;\{\underbrace{J_{1} \ldots, J_{j}, \ldots}_{m}\}\right] \\
& -\sum_{j}^{m} \operatorname{Slice}[J_{j} ; \underbrace{\left\{\ldots, I_{i}, \ldots\right\}}_{n}\} ; \underbrace{\ldots, J_{k \neq j}, \ldots}_{m-1}\}] .
\end{aligned}
$$

From Eqs. (2)-(11) is evident that by evaluating the Slice function we are able to evaluate any arbitrary combination of unions, intersections, and differences between fused spheres.

\section{CONSTRUCTING A DISPLACEMENT MOVE FOR THE SW SYSTEM, BASED ON THE UNION-INTERSECTIONS-DIFFERENCES ALGORITHM}

A crucial entity in the proposed sampling scheme is the locus of points where a possible placement of a square-well (SW) particle $\alpha$ will result in a specified interaction energy with the rest of the SW particles of the system. The volume of all points that result to the same energy level is calculated as follows.

Starting from the old state we randomly select the particle $\alpha$ to be displaced. In contrast with the traditional displacement move, our displacement is not centered on the old position of the displaced particle but in a randomly selected point within a box centered on the old position. Such a selection of a trial box ensures that the displacement move satisfies the detailed balance criteria as described in Appendix A, by equalizing the probability of selecting the same trial box from the old and the new state.

Since the new position of the particle could be any point within the trial box, we should consider interaction of the displaced particle with all particles whose distance from the center of the trial box is smaller than the interaction distance plus half the body-diagonal of the trial box.

For each particle $i$ in the volume of interest, except the displaced one, two spheres are considered. Both spheres have the same center as particle $i$. The first sphere ("exclusion" sphere, $s_{i}^{\text {excl }}$ ) has a radius equal to the sum of hard-core radii of the original particle $i$ and the displaced particle $\alpha$, as defined in Eq. (12). Whereas the second sphere ("interaction" sphere, $\left.S_{i}^{\text {inter }}\right)$ has a radius equal to the previous radius plus the width of the attractive well defined in Eq. (13).

$$
\begin{aligned}
& R_{i}^{\mathrm{excl}}=\frac{\sigma_{\alpha}+\sigma_{i}}{2}, \\
& R_{i}^{\mathrm{inter}}=\frac{\lambda \sigma_{\alpha}+\lambda \sigma_{i}}{2},
\end{aligned}
$$

where $\lambda \equiv(1+\delta)$. Any attempt to move particle $\alpha$ to a point inside an exclusion sphere will result in an infinite interaction with the particle $i$. On the other hand moving particle $\alpha$ to a point that belongs to the interaction sphere but not to the exclusion sphere will result in an attractive interaction with this particle. Finally, insertion of the particle $\alpha$ in any other point of the test volume will result in no interaction between particles $\alpha$ and $i$. Therefore the number of interactions, and hence the interaction energy, for a given trial position of particle $\alpha$, is determined by the number of distinct interaction spheres to which this point belongs. The volume of points resulting in only one interaction between the displaced particle and the rest of the system is given by

$$
V_{1}=\sum_{i=1}^{N}\left[V_{\left(S_{i}^{\text {inter }}-\left(s_{i}^{\text {excl }} \cup \ldots \cup S_{j \neq i}^{\text {inter }} \cup \ldots\right)\right) \cap B}\right],
$$

where $B$ is the volume where the displacement of particle $\alpha$ is attempted (the volume of the trial box in our case), $N$ is the number of particles in the system for which $S_{i}^{\text {inter }} \cap B \neq 0$ (i.e., there are points in volume $B$ where placing particle $\alpha$ will result in interaction or overlap with particle $i$ ), and $j$ runs on the subset of those particles for which at the same time $\left(S_{j}^{\text {inter }} \cap S_{i}^{\text {inter }}\right) \cap B \neq \varnothing$ defined as neighbors to particle $i$. (i.e., there may be points in volume $B$ where placing particle $\alpha$ will result in interaction with particles $i$ and $j$ ). The incorporation of any other particle, that does not satisfy the above conditions have no contribution, on the other hand may increase dramatically the computational cost.

Similarly the volume of points that will result in two interactions is given by

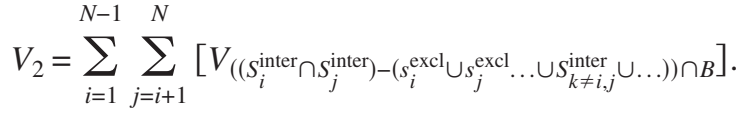

For the general case of $n$ interactions the corresponding volume is calculated as the sum of all possible combinations of the difference between the intersection of $n$ interaction spheres $\left(S_{i_{\xi}}^{\text {inter }}\right)$ of particle $i_{\xi}, \xi \in[1, n]$ and the union of the corresponding exclusion spheres $s_{i_{\xi}}^{\text {excl }}$ and the $m$ common neighboring interaction sphere, $\left(S_{k}^{\text {inter }}\right)$. The corresponding 
expressions for $V_{n}$ with $n=2,3, \ldots$, are rather unwieldy and are given in Appendix B.

Having evaluated the volume of all possible levels $V_{n}$ we select to place the displaced particle to one of them according to the Boltzmann weighted probability given by

$$
P(n)=\frac{V_{n}^{*} e^{-\beta(-n)}}{\sum_{i=0}^{12} V_{i}^{*} e^{-\beta(-i)}} .
$$

Having selected the energy level according to Eq. (16), we have to select one of the combinations of $n$ particles with which the displaced particle should interact. In order to do so we keep a list of all nonzero volumes $V(I, n)$ for our calculation and select one of them given by

$$
P(I, n)=\frac{V(I, n)}{\sum V(I, n)} .
$$

For each one of the $n$ selected particles the boxes that bound the interaction spheres of those particles are defined. Trial insertions of the displaced molecule are attempted in the box formed by the intersection of the interaction boxes and the initial trial volume. The first trial position that satisfies the condition of interacting with only the $n$ selected particles is selected as the new position for the displaced particle. In Appendix A we demonstrate that the proposed scheme satisfies detailed balance. In Appendix B we briefly summarize the procedure to calculate the volume of all possible levels $\left(V_{n}\right)$.

\section{SIMULATION DETAILS}

Several numerical studies have been performed on the $\mathrm{SW}$ model in the past. ${ }^{21-24}$ The parameters $\varepsilon, \delta$, and $\sigma$ determine the phase diagram of the system. Because of the corresponding-states principle, all systems with the same value of $(\delta / \sigma)$ have equivalent phase diagrams. By changing the range of interaction $\delta$, we can go from the van der Waals limit $(\delta=\infty)$ to the adhesive sphere limit $(\delta=0)$. Most of the previous simulation studies focused on square-well widths that were considered to be typical for simple liquids. ${ }^{21-24}$ However, in order to model complex liquids (colloids and protein solutions), it is important to have efficient schemes to simulate SW fluids with short-ranged attraction. With conventional MC schemes, it is, however, difficult to equilibrate systems at the conditions of vapor liquid equilibrium for values of $\delta<0.125$. Below, we show that, with the present method, we can sample systems with very small values of $\delta$.

In our approach we generate a Markov chain of configurations by exhaustive enumeration of all possible interaction states in the vicinity of the old configuration. We always accept one of these trial states according to its Boltzmann weight. To this end, we employ the scheme described above. For the case of a one-component system, we refer to the interaction energy level of the displaced particle as the number of interactions of that particle $i$ with the rest of the system $\left(\sum_{j \neq i}^{N} u_{i j} /-\varepsilon\right)$.

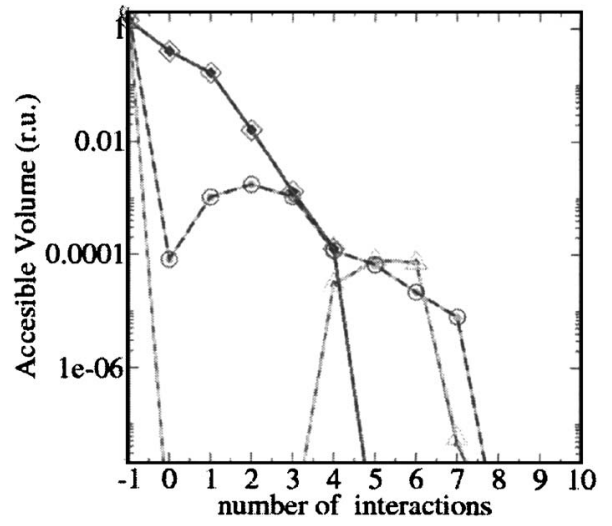

FIG. 2. Comparison between the analytical evaluation of the accessible volume for the insertion of a SW particle in a specific interaction levels in a box of length $1.251 \sigma$, using the proposed analytical method (filled symbols) and $500 \times 10^{6}$ trial "ghost" insertions (open symbols). Three attempted moves are shown for the displacement of a SW particle in a system of 108 SW particles of $\lambda=1.05, T^{*}=0.4$, and $\rho^{*}=0.723$. Interaction level -1 represents the case where the placement of a SW particle will result in an overlap.

\section{RESULTS AND DISCUSSION}

As a first test, we used the analytical evaluation of the volumes that correspond to $n$-particle interactions, to compute the excess chemical potential of a square-well fluid, using a modification of the Widom particle-insertion method. ${ }^{25}$ In the normal Widom scheme, the excess chemical potential is estimated by performing a large number of virtual trial insertions of a test particle in a given volume of the fluid. The chemical potential is then computed using

$$
\mu_{\mathrm{ex}}=-k_{B} T \ln \left\langle e^{-\beta \Delta U}\right\rangle,
$$

where $\Delta U$ is the potential energy of the test particle at its randomly chosen test position. With the present scheme we can do better, because for any given subvolume $V$, and for any configuration of the system, we can estimate the contribution to $\langle\exp (-\beta \Delta U)\rangle$,

$$
\overline{e^{-\beta \Delta U}}=\sum_{n=0}^{12}\left(\frac{V_{n}}{V}\right) e^{n \beta \varepsilon} .
$$

This method works well, even for very short-ranged attractions, where the volumes $V_{n}$ become very small for larger $n$. Rather than comparing the result averaged over many configurations of the system, we compared the analytical result for a few specific configurations with the result that was obtained with the conventional scheme, using $500 \times 10^{6}$ trial insertions. For both schemes, we considered the same test volume with a size of $1.96 \sigma^{3}$. The three configurations used for the comparison of the accessible volumes presented in Fig. 2 were generated at $T^{*}=0.4$ and $\rho^{*}=0.723$ for a system of $108 \mathrm{SW}$ particles with $\delta=0.05$. The computational cost for the $500 \times 10^{6}$ trial insertions $\left(t_{\text {ins }}\right)$ is three orders of magnitude higher than the computational cost $\left(t_{a}\right)$ of the exact analytical solution. We are not proposing to use the present scheme as a general method to compute chemical potentials because the computation of $\langle\exp (-\beta \Delta U)\rangle$ will require sampling many configurations using as test volume the total volume of the system and not a subvolume as in our test case 


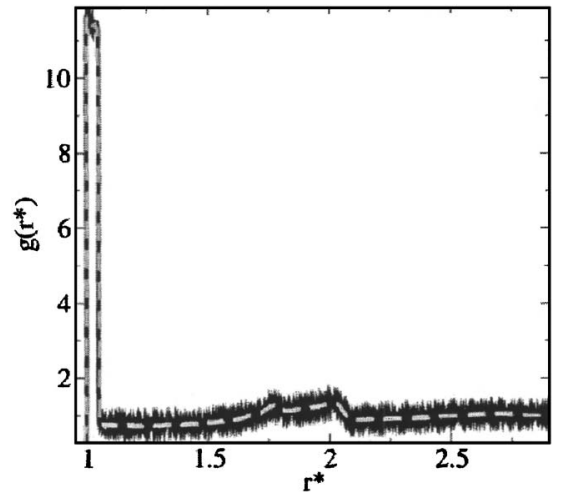

FIG. 3. Radial distribution function $g\left(r^{*}\right)$ of a system of $108 \mathrm{SW}$ particles of $\lambda=1.05$ at $T^{*}=0.37$ and $\rho^{*}=0.542$ sampled with the proposed sampling (broken line) and the traditional random displacement method (continuum line). The difference in the noise of the two graphs is due to the use of different binning size (for visibility reasons) in the two simulations.

and then the advantage of the analytical method is lost (except for $\delta \ll 1)$.

In Figs. 3-5 we show that the proposed algorithm yields the same results as the conventional Metropolis sampling, in a regime where we can still use the latter. The simulations were performed in the canonical (constant-NVT) ensemble and all results are presented in reduced units. ${ }^{26} \mathrm{We}$ considered a system of $108 \mathrm{SW}$ particles with $\delta=0.05, T^{*}=0.37$, and $\rho^{*}=0.542$. At this temperature and density to guarantee the conventional MC scheme still yields reliable results to which we can compare our results. In Fig. 3 we show a comparison of the radial distribution function $g(r)$ as computed with the present scheme and with the standard MC method. As can be seen in the figure, the present method reproduces the results of the conventional $\mathrm{MC}$ scheme. In Fig. 4, we used the two schemes to compute energy histograms for the same model system. Again, we find good agreement with conventional MC sampling.

The advantage of our analytical evaluation of the interaction volumes over the use of trial insertions is not only that, for a given configuration of the particles, we obtain the exact answer but also that it sometimes leads to drastic savings in CPU time. This is the case when the interaction vol-

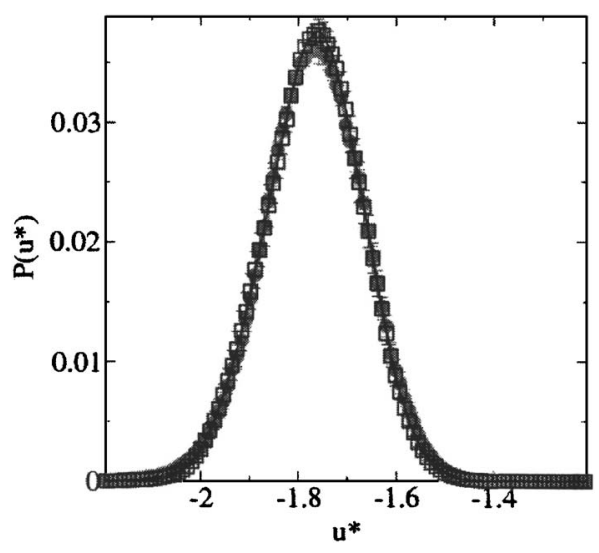

FIG. 4. Probability density of the internal energy per particle in a system of $108 \mathrm{SW}$ particles of $\lambda=1.05$ at $T^{*}=0.4$ and $\rho^{*}=0.542$ sampled with the proposed sampling (filled circles) and the traditional random displacement method (open squares).

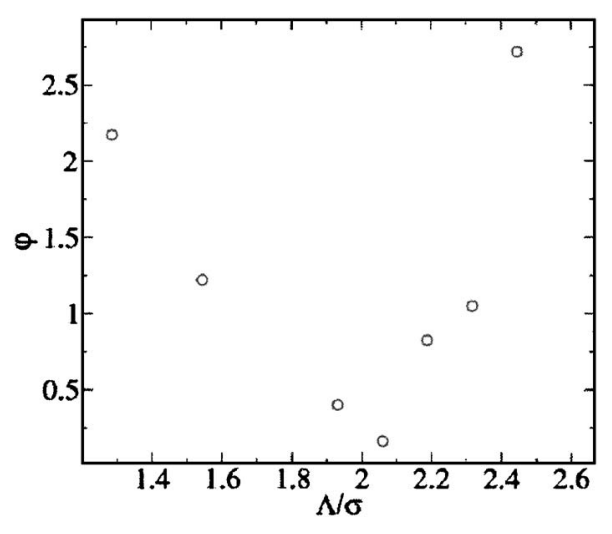

FIG. 5. Computational efficiency in a system of 108 SW particles of $\lambda$ $=1.005$ at $T^{*}=0.225$ and $\rho^{*}=0.663$ of the proposed sampling method and the traditional conventional Metropolis sampling $\left(\varphi=s^{2} t / s_{\text {Met }}^{2} t_{\text {Met }}\right)$ as a function of displacement trial box length $(\Lambda)$ where the displacement is performed, see text.

ume is small, as is the case for SW particles with shortranged interactions. The advantage is particularly pronounced at low temperatures.

Whether or not the present scheme advantageous depends strongly on the simulation conditions. The reason is that, in contrast with the usual MC method, the CPU cost of the proposed algorithm depends strongly on the average energy of the system and on the size of the trial box where the insertions are performed. Depending on the conditions, one step of the proposed method may be equivalent to hundreds of random displacement moves. Figure 5 shows an illustration of the computational efficiency of the present scheme for a system of $108 \mathrm{SW}$ particles with $\delta=0.005, T^{*}=0.225$, and $\rho^{*}=0.6625$. Figure 5 compares the computational efficiency in terms of the mean square of the statistical error $s$ of the potential energy times the CPU consumption $\left(s^{2} t_{\mathrm{CPU}}\right)$, divided by the efficiency of conventional MC sampling $\varphi$ $=s^{2} t / s_{\text {Met }}^{2} t_{\text {Met }}$, where the maximum displacement length was adjusted during the equilibration period in order to yield $\sim 30 \%$ acceptance. The statistical uncertainty $s$ of the potential energy was estimated from the variance in the block averages. Concluding we argue that with the appropriate choice of parameters (mainly the size of the displacement box $\Lambda$ ) the proposed method became much more efficient than the random displacement in low temperatures and higher densities. The figure shows that with an appropriate choice of parameters (in particular, the size of the displacement box) the proposed method can be much more efficient than random sampling, in particular, at low temperatures and high densities. These conditions are relevant for the study of (metastable) liquid-liquid equilibria. In a separate publication we show how the efficiency of the present scheme can be increased even more by combining it with a scheme that includes the contributions of rejected trial moves in the sampling of averages. ${ }^{27}$

\section{ACKNOWLEDGMENTS}

One of the authors (G.B.) would like to thank Professor D. N. Theodorou for providing him with the code for the analytical evaluation of the volume of fused spheres, and for 
helpful discussions. This work is part of the research program of the "Stichting voor Fundamenteel Onderzoek der Materie (FOM)," which is financially supported by the "Nederlandse Organisatie voor Wetenschappelijk Onderzoek (NWO)." The support by European Community through a Marie Curie individual Fellowship with Contract No. HPMF-CT-2002-02089 is greatly acknowledged. In addition, this work was supported through EU Contract No. MRTNCT-2003-504712.

\section{APPENDIX A: DETAILED BALANCE DERIVATION}

The detailed balance condition implies that the following equation should be satisfied in any attempt to move from the old state $o$ to the new state $n:{ }^{26}$

$$
\begin{aligned}
\mathcal{N}(o) & \times \alpha(o \rightarrow n) \times \operatorname{acc}(o \rightarrow n) \\
= & \mathcal{N}(n) \times \alpha(n \rightarrow o) \times \operatorname{acc}(n \rightarrow o),
\end{aligned}
$$

where $\mathcal{N}(i)$ is proportional to the probability of finding the system in state $i, \alpha(i \rightarrow j)$ is the probability of attempting to go from state $i$ to state $j$ and is usually referred to as the underlying matrix of the Markov chain, and $\operatorname{acc}(i \rightarrow j)$ is the probability of accepting the move from state $i$ to state $j$. In the original Metropolis scheme, $\alpha$ is chosen to be symmetrical whereas the acceptance criteria are chosen in a way to satisfy Eq. (A1). The proposed scheme is based on contracting the $\alpha(i \rightarrow j)$ in a way that the resulting acceptance probability $\operatorname{acc}(i \rightarrow j)$ is always 1 . From Eq. (A1) it follows that the above statement can be expressed as

$$
\frac{\alpha(o \rightarrow n)}{\alpha(n \rightarrow o)}=\frac{\mathcal{N}(n)}{\mathcal{N}(o)} \text {. }
$$

In the case of the canonical ensemble the right-hand side of Eq. (A2) results on the ratio of the Boltzmann factors of the new and old states, where $U$ is the potential energy of the system on $N$ particles, and $u$ is the interaction energy of the displaced particle with the rest (in reduced units for onecomponent system), $-u$ is equal to the number of interactions that the displaced particle has (i.e., number of neighbors whose center-to-center distance is closer than $\lambda \sigma$ ).

$$
\frac{\mathcal{N}(n)}{\mathcal{N}(o)}=\frac{e^{-\beta U(n)}}{e^{-\beta U(o)}}=e^{-\beta(U(n)-U(o))}=e^{-\beta(u(n)-u(o))} .
$$

The proposed displacement moves consist of the following steps:

(a) First the particle that is about to move is selected at random over the $N$ possible particles. A box of volume $V_{\text {test }}=\Lambda^{3}$ and center at the center of the old position of the displaced particle is considered.

(b) Within this volume a point is selected at random in order to be the center of a new box with equal volume. In this volume the analytical calculation described in the text is performed resulting to the volumes of points that potentially can result in any given number of interactions between the displaced particle and the $N-1$ particles in the system.

(c) As discussed in the text we choose one of those levels according to Eq. (16). (d) Finally we chose one combination (I) of interaction particles according to Eq. (17).

(e) We evaluate the parallelepiped box that forms from the union of the boxes enscribing those particles and select the first point in this parallelepiped box that satisfies the condition of belonging to the preselected energy level interaction with the combination $(I)$ from step (d).

According to the above procedure the underlying matrix of the Markov chain $\alpha$ is given by the following:

$$
\begin{aligned}
\alpha(o \rightarrow n) & =\frac{1}{N} \times \frac{1}{V_{\text {test }}} \times \frac{V_{n}^{*} e^{-\beta u(n)}}{\sum_{i=0}^{\infty} V_{i}^{*} e^{-\beta u(i)}} \times \frac{V\left(I_{n}, n\right)}{V_{n}} \\
& \times \frac{1}{V\left(I_{n}, n\right)}, \\
\alpha(n \rightarrow o) & =\frac{1}{N} \times \frac{1}{V_{\text {test }}} \times \frac{V_{o}^{*} e^{-\beta u(o)}}{\sum_{i=0}^{\infty} V_{i}^{*} e^{-\beta u(i)}} \times \frac{V\left(I_{o}, o\right)}{V_{o}} \\
\times & \frac{1}{V\left(I_{o}, o\right)} .
\end{aligned}
$$

From Eqs. (A3), (A4a), and (A4b) it follows (A5) thus (A2) is satisfied and so forth the proposed scheme obeys the condition of (super) detailed balance condition.

$$
\frac{\alpha(o \rightarrow n)}{\alpha(n \rightarrow o)}=\frac{e^{-\beta u(n)} / \sum_{i=0}^{\infty} V_{i}^{*} e^{-\beta u(i)}}{e^{-\beta u(o)} / \sum_{i=0}^{\infty} V_{i}^{*} e^{-\beta u(i)}}=e^{-\beta(u(n)-u(o))}=\frac{\mathcal{N}(n)}{\mathcal{N}(o)}
$$

Note that since the volumes of different energy levels are a function of the trial box, step (b) is crucial in satisfying that the volumes in the forward and the reverse move correspond to the same box and therefore Eq. (A5) is valid.

\section{APPENDIX B: PROCEDURE TO CALCULATE THE VOLUMES OF ALL INTERACTION LEVELS}

The magnitude of the volume where a test interacts with $n$ other particles is calculated as the sum of all possible combinations of the difference between the intersection of $n$ interaction spheres $\left(S_{i_{\xi}}^{\text {inter }}\right)$ of particle $i_{\xi}, \xi \in[1, n]$ and the union of the corresponding exclusion spheres $s_{i_{\xi}}^{\text {excl }}$ and the $m$ common neighboring interaction sphere $\left(S_{k}^{\text {inter }}\right)$, where a particle $k$ has nonzero contribution in the calculation only if it is "neighbor" to all particles $i_{\xi}, \forall \xi \in[1, n]$ particles thus satisfy the following:

$$
\left(\left(\bigcap_{\xi=1}^{n} S_{i_{\xi}}^{\text {inter }}\right) \cap S_{k}^{\text {inter }}\right) \cap B \neq \varnothing
$$




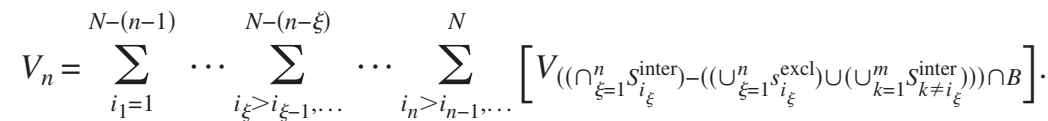

Taking into account that the exclusion spheres are completely embedded in the corresponding interaction spheres, the accumulated volume of Eq. (B2) can be rewritten based on Eq. (11) as

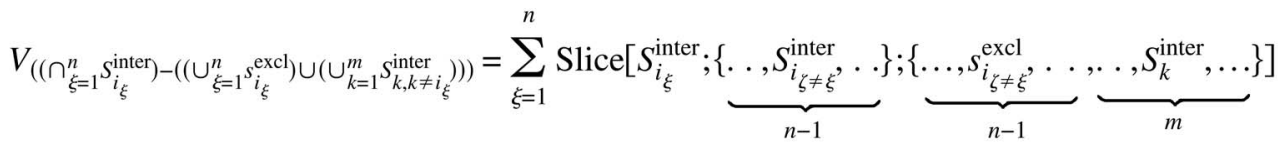

$$
\begin{aligned}
& -\sum_{\xi}^{n} \operatorname{Slice}[s_{i_{\xi}}^{\text {excl }} ; \underbrace{\left\{\ldots, S_{i_{\xi \neq \xi}}^{\text {inter }}, \ldots\right\}}_{n-1} ; \underbrace{\ldots, s_{i_{\xi \neq \xi}}^{\text {excl }}, \ldots, \underbrace{\text { inter }}_{m}, \ldots\}}_{n-1}] \\
& -\sum_{k=1}^{m} \operatorname{Slice}[S_{k}^{\text {inter }} ;\{\underbrace{\left\{, S_{i_{\xi}}^{\text {inter }}, \ldots\right.}_{n}\} ; \underbrace{\left\{\ldots, s_{i_{\xi}}^{\text {excl }}, \ldots,\right.}_{n} \underbrace{\left.\ldots, S_{i \neq k}^{\text {inter }}, \ldots\right\}}_{m-1}] \text {, }
\end{aligned}
$$

where $i_{\xi}(1 \leqslant \xi \leqslant n)$ denotes one of the $n$ particles with which the displaced particle will interact in the volume given by Eq. (B3). For a given $i_{\xi}$, the index $\zeta$ denotes one of the remaining $n-1$ particles with $\zeta \neq \xi$.

In Fig. 6 we present a schematic representation of $V_{3}$ in a two-dimensional test case. It should be noted that, for a given energy level $n$, terms from the first sum on the righthand side of Eq. (B3) are frequently identical to terms of the third sum of the next energy level $n+1$. By the appropriate bookkeeping of the calculated volumes the computational cost is greatly reduced.

It is possible to further reduce the computational cost, if in Eq. (B3), one substitutes the exclusion radius instead of the "interacting" (i.e., substituting $S_{k}^{\text {inter }}$ with $s_{k}^{\text {excl }}$ ), for the $m$ "neighboring" spheres then the resulting volume is the volume that can lead to at least $n$ interactions. We use this information in the case of $n=1$ to find the total volume that a given sphere can contribute to all energy levels. In the pro-

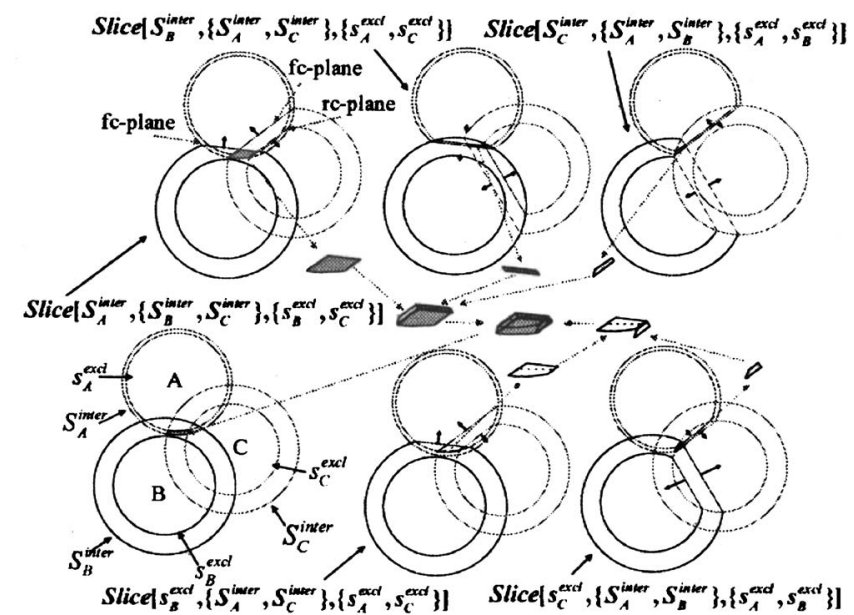

FIG. 6. A schematic representation of volume $\left(V_{3}\right)$ of points where the placement of an additional particle will result in three interactions (with particles $A, B$, and $D$ as is evaluated by Eq. (B3) in a two-dimensional test. For the notation see text. cess of our calculation we accumulate the volume of each sphere to a given energy level. When the cumulative volume reaches the volume described above, this sphere can appear only as a neighboring sphere. We note that the order of investigating the different energy levels has a significant effect on the computational cost.

Below, we briefly summarize the procedure to calculate the volume corresponding to $n$ interactions according to Eq. (B2):

(1) First we identify and label the particles for which the intersection of the interaction sphere and the box of interest may be nonzero $\left(S_{i}^{\text {inter }} \cap B \neq 0\right)$.

(2) The next step is to find all possible combinations of particles that may result in the desirable number of interactions $n$. In order to do so we loop recursively over all labeled particles, and find the combinations that result in a cluster of at least $n$ particles that satisfy the condition that the center-to-center distance between each particle and each of the $i$ particle is less than the sum of the interacting radius of the two particles. This condition is less strict than Eq. (B1). Since it is much cheaper than the condition of Eq. (B1) it is applied in an early state, in order to reduce the overall number of particles used in the calculation. Multiple counting is avoided by appropriate bookkeeping. At this point we have came up with a list of combinations of $n$ particles and their $m$ "neighbors" that may contribute to the volume of interest. Note that only a very small part of them will result in nonzero contribution.

(3) For every combination in the above list the contribution to the sum of Eq. (B2) is evaluated according to Eq. (B3).

For each one of the $n$ particles $i$ the first two terms of Eq. (B3) are being evaluated.

(a) The first term of Eq. (B3) requires that the sphere $i$ has 
the interacting radius $R_{i}^{\text {inter }}$ defined by Eq. (13), and has been sliced by

i. $n-1$, FC planes, formed by the intersection of sphere $S_{i}^{\text {inter }}$ and the $n-1$ spheres in the list \{having also their interacting radius defined by Eq. (13) $\left.S_{j}^{\text {inter }}, \forall j \in[1, n] \neq i\right\}$.

ii. $n-1 \mathrm{RM}$ planes, formed by the intersection of sphere $S_{i}^{\text {inter }}$ and the $n-1$ spheres in the list \{having their exclusion radius defined by Eq. (12) $s_{j}^{\text {excl }}$, $\forall j \in[1, n] \neq i\}$.

iii. $m, \mathrm{RM}$ planes, formed by the intersection of sphere $S_{i}^{\text {inter }}$ and the $m$ neighboring spheres in the list \{having their interacting radius defined by Eq. (13) $\left.S_{k}^{\text {inter }}, \forall k \in[1, m]\right\}$.

(b) The second term of Eq. (B3) requires that the sphere $s_{i}^{\text {excl }}$ has been sliced by

i. $n-1$, FC planes, formed by the intersection of sphere $s_{i}^{\text {excl }}$ and the $n-1$ spheres in the list having also the interacting radius defined by Eq. (13) $S_{j}^{\text {inter }}$, $\forall j \in[1, n] \neq i\}$.

ii. $n-1, \mathrm{RM}$ planes, formed by the intersection of sphere $s_{i}^{\text {excl }}$ and the $n-1$ spheres in the list \{having the exclusion radius defined by Eq. (12) $s_{j}^{\text {excl }}, \forall j$ $\in[1, n] \neq i\}$.

iii. $m, \mathrm{RM}$ planes, formed by the intersection of sphere $s_{i}^{\text {excl }}$ and the $m$ neighboring spheres in the list $\{$ having the interacting radius defined by Eq. (13) $S_{k}^{\text {inter }}$, $\forall k \in[1, m]\}$.

(c) The third term of Eq. (B3) has to be evaluated for each one of the $m$ neighboring spheres $S_{k}^{\text {inter }}$. This term requires that the sphere $S_{k}^{\text {inter }}$ has the interacting radius $R_{i}^{\text {inter }}$ defined by Eq. (13), and has been sliced by

i. $n$, FC planes, formed by the intersection of sphere $S_{k}^{\text {inter }}$ and the $n$ spheres in the list \{having also the interacting radius defined by Eq. (13), $S_{j}^{\text {inter }}, \forall j$ $\in[1, n]\}$.

ii. $\quad n, \mathrm{RM}$ planes, formed by the intersection of sphere $S_{k}^{\text {inter }}$ and the $n$ spheres in the list having the exclusion radius defined by Eq. (12), $s_{j}^{\text {excl }}, \forall j$ $\in[1, n]\}$.

iii. $m-1, \mathrm{RM}$ planes, formed by the intersection of sphere $S_{k}^{\text {inter }}$ and the $m-1$ neighboring spheres in the list \{having the interacting radius defined by Eq. (12) $\left.S_{j}^{\text {inter }}, \forall j \in[1, n]\right\}$.

In order to speed up the calculations the first term is evaluated. If it turns out to be zero then no further calculation is required for this combination of $n$ particles since the other two terms have to be also zero. Confining the calculation to a box requires that all spheres should be sliced by the boundary planes of the box. This procedure is followed for all energy levels $n$ from 1 to 14 , (or more efficiently from 14 to 1) and the volume of points that correspond to no interaction is calculated from the difference between the total volume of the box and the volume of all energy levels. We used as a maximum energy levels 14 since for infinitely short-ranged interactions we know that the maximum value is 12 .

${ }^{1}$ W. G. T. Kranendonk and D. Frenkel, Mol. Phys. 64, 403 (1988).

${ }^{2}$ D. A. Young and B. J. Alder, J. Chem. Phys. 73, 2430 (1980).

${ }^{3}$ D. A. Young and B. J. Alder, J. Chem. Phys. 70, 473 (1978).

${ }^{4}$ B. J. Alder, D. A. Young, and M. A. Mark, J. Chem. Phys. 56, 3013 (1972).

${ }^{5}$ G. A. Vliegenthart, J. F. M. Lodge, and H. N. W. Lekkerkerker, Physica A 263, 378 (1999).

${ }^{6}$ M. Miller and D. Frenkel, Phys. Rev. Lett. 90, 135702 (2003).

${ }^{7}$ M. J. Hagen and D. Frenkel, J. Chem. Phys. 101, 4093 (1994).

${ }^{8}$ N. Asherie, A. Lomakin, and G. B. Benedek, Phys. Rev. Lett. 77, 4832 (1996).

${ }^{9}$ D. Rosenbaum, P. C. Zamora, and C. F. Zukoski, Phys. Rev. Lett. 76, 150 (1996).

${ }^{10}$ P. R. ten Wolde and D. Frenkel, Science 277, 1975 (1997).

${ }^{11}$ L. Onsager, Chem. Rev. (Washington, D.C.) 13, 73 (1933).

${ }^{12}$ W. G. McMillan and J. E. Mayer, J. Chem. Phys. 13, 276 (1945).

${ }^{13}$ C. Ishimoto and T. Tanaka, Phys. Rev. Lett. 39, 474 (1977).

${ }^{14}$ O. Galkin and P. G. Vekilov, Proc. Natl. Acad. Sci. U.S.A. 97, 6277 (2000).

${ }^{15}$ M. Muschol and F. Rosenberger, J. Chem. Phys. 107, 1953 (1997).

${ }^{16}$ V. Talanquer and D. W. Oxtoby, J. Chem. Phys. 109, 223 (1998).

${ }^{17}$ R. Sear, Phys. Rev. E 63, 066105 (2001); J. Chem. Phys. 114, 3170 (2001).

${ }^{18}$ B. Chen and J. I. Siepmann, J. Phys. Chem. B 104, 8725 (2000).

${ }^{19}$ S. Wierzchowski and D. A. Kofke, J. Chem. Phys. 114, 8752 (2001).

${ }^{20}$ L. R. Dod and D. N. Theodorou, Mol. Phys. 72, 1313 (1991).

${ }^{21}$ L. Vega, E. de Miguel, and L. F. Rull, J. Chem. Phys. 96, 2296 (1992).

${ }^{22}$ F. Del Rio, E.Avalos, R. Espindola, L. F. Rull, G. Jackson, and S. Lago, Mol. Phys. 100, 2531 (2002).

${ }^{23}$ N. V. Brilliantov and J. P. Valleau, J. Chem. Phys. 108, 1123 (1998).

${ }^{24}$ J. R. Elliott and L. Hu, J. Chem. Phys. 110, 3043 (1999).

${ }^{25}$ B. Widom, J. Chem. Phys. 39, 2808 (1963).

${ }^{26}$ D. Frenkel and B. Smit, Understanding Molecular Simulation: From Algorithms to Applications, 2nd ed. (Academic, Boston, 2002).

${ }^{27}$ G. C. Boulougouris and D. Frenkel, J. Theor. Comput. Chem. 1, 389 (2005). 